\title{
Tumor fibroso maligno de pleura
}

\author{
Malignant fibrous tumor of the pleura
}

\author{
Laura Marcela Fino, Jorge Alberto Carrillo \\ - Bogotá, D.C. (Colombia)
}

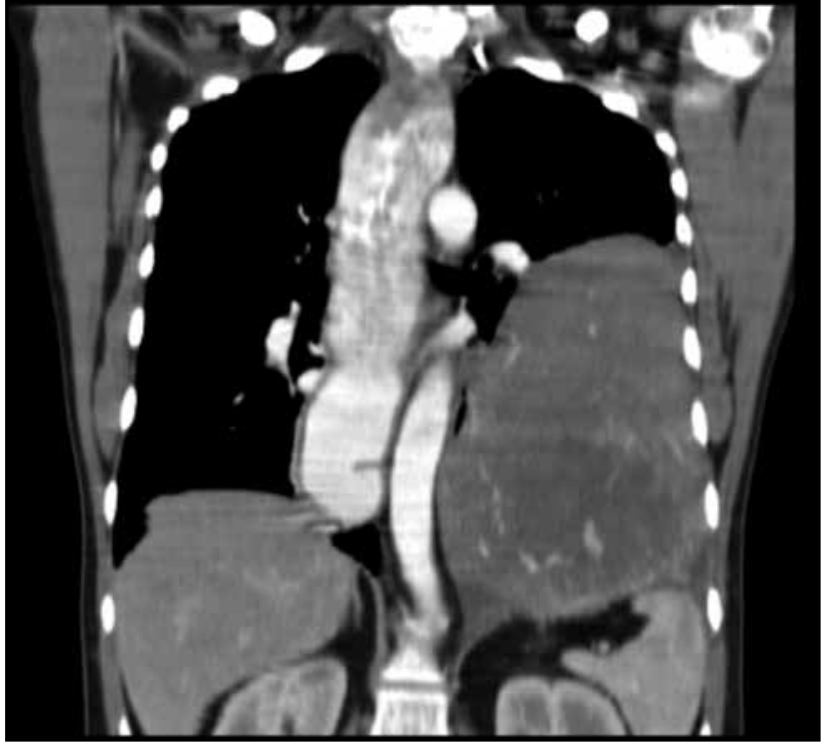

Figura 1. Tomografía evidencia masa pleural.

Varón de 50 años con disnea, tos, pérdida de peso, mialgias y poliartralgias de nueve meses de evolución.

La tomografía computarizada (TC) evidenció masa pleural, sólida, de densidad heterogénea, vascularizada, con diámetros mayores de $18 \times 16 \mathrm{~cm}$, asociada a líquido pleural libre, atelectasia del pulmón adyacente y dilatación del esófago en toda su extensión, como hallazgo incidental (Figura 1).

El estudio de la masa resecada (Figura 2) demostró pleomorfismo celular, Ki-67 60\%, áreas necróticas, numerosas mitosis, positividad para BCL2 y CD34, y negatividad para S100 y vimentina. Se diagnosticó tumor fibroso solitario de pleura maligno.

El tumor fibroso solitario de pleura es una neoplasia rara, generalmente benigna. El 10\% de TFSP son potencialmente malignos y pueden cursar con recurrencia local y metástasis (1-3).

El comportamiento maligno se asocia a factores pronósticos que incluyen: elevada actividad mitótica, necrosis, bordes quirúrgicos positivos y un diámetro tumoral mayor de $10 \mathrm{~cm}(1,4,5)$.

\section{Referencias}

1. Tapias LF, Mino-Kenudson M, Lee H, Wright C, Gaissert HA, Wain JC, et al. Risk factor analysis for the recurrence of resected solitary fibrous tumours of the pleura: A 33-year experience and proposal for a scoring system. Eur J Cardiothoracic Surg. 2013; 44 (1): 111-7.

2. Ricciuti B, Metro G, Leonardi GC, Sordo R del, Colella R, Puma F, et al.

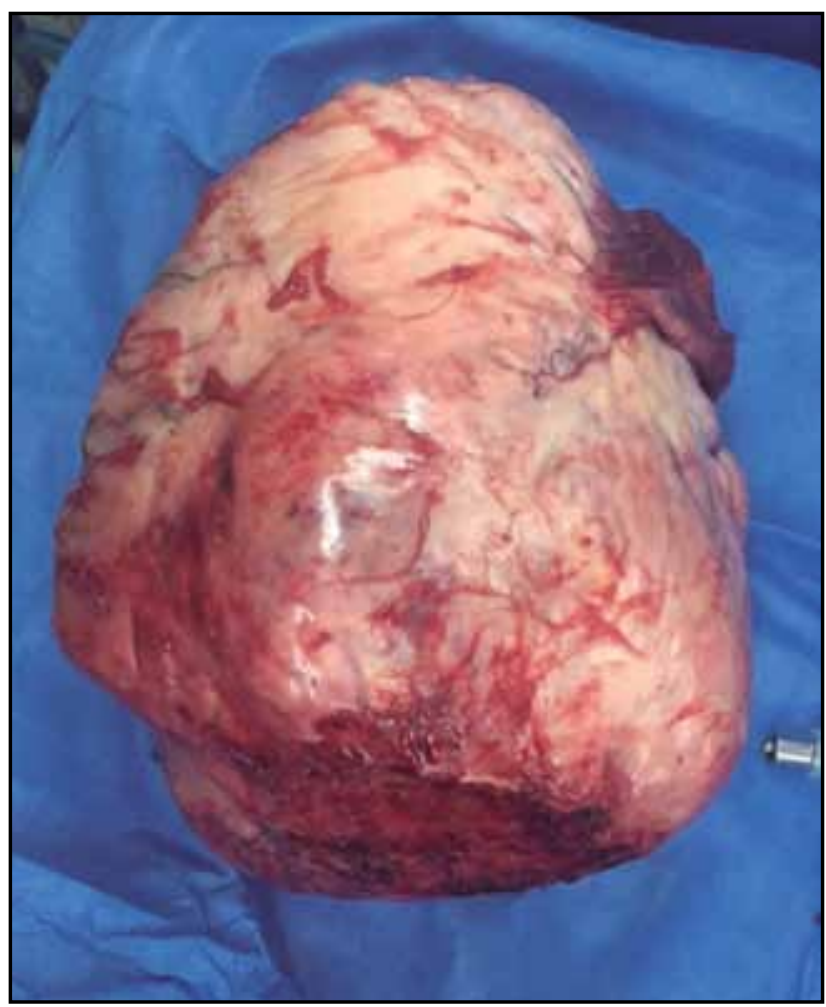

Figura 2. Anatomía patológica masa resecada.

Malignant giant solitary fibrous tumor of the pleura metastatic to the thyroid gland. Tumori [Internet]. 2016; 102 (Suppl. 2): 16-21. Available from: http:// www.tumorijournal.com/article/93517541-0d16-499c-8777-a18dcae33904.

3. Luján M, Mejía S, Rojas S y Mira S. Síndrome de Doege Potter Tumor fibroso de pleura irresecable asociado a hipoglucemia. Acta Med Colomb. 2009; 34 (4): $185-8$.

4. Van Houdt WJ, Westerveld CMA, Vrijenhoek JEP, van Gorp J, van Coevorden F, Verhoef C, et al. Prognosis of solitary fibrous tumors: a multicenter study. Ann Surg Oncol [Internet]. 2013; 20 (13): 4090-5. Available from: http://www. ncbi.nlm.nih.gov/pubmed/24052313

5. León F, Bannura F, Solovera ME y Salas P. Tumor fibroso solitario de la pleura asociado a síndromes paraneoplásicos. Rev Chilena Cirug. 2013; (65): 255-9.

Dra. Laura Marcela Fino Velásquez: Universidad del Rosario; Dr. Jorge Alberto Carrillo Bayona: Hospital Universitario Mayor (HUM) Méderi, Universidad del Rosario. Bogotá, D.C. (Colombia).

Correspondencia: Dra. Laura Marcela Fino Velásquez. Bogotá, D.C. (Colombia). E-mail: lauramarcelafino@gmail.com

Recibido: 27/VII/2017 Aceptado: 10/IV/2018 